\title{
Contributo all'identificazione di manoscritti francesi della Biblioteca Nazionale Universitaria di Torino
}

\section{Angelo Giaccaria}

\section{(2) OpenEdition}

1 Journals

\section{Edizione digitale}

URL: http://journals.openedition.org/studifrancesi/9734

DOI: 10.4000/studifrancesi.9734

ISSN: 2421-5856

\section{Editore}

Rosenberg \& Sellier

\section{Edizione cartacea}

Data di pubblicazione: 1 octobre 2007

Paginazione: 335-353

ISSN: 0039-2944

\section{Notizia bibliografica digitale}

Angelo Giaccaria, «Contributo all'identificazione di manoscritti francesi della Biblioteca Nazionale Universitaria di Torino», Studi Francesi [Online], 152 (LI | II) | 2007, online dal 30 novembre 2015, consultato il 09 janvier 2021. URL: http://journals.openedition.org/studifrancesi/9734 ; DOI: https:// doi.org/10.4000/studifrancesi.9734

\section{(c)}

Studi Francesi è distribuita con Licenza Creative Commons Attribuzione - Non commerciale - Non opere derivate 4.0 Internazionale. 


\section{Contributo all'identificazione di manoscritti francesi della Biblioteca Nazionale Universitaria di Torino*}

Nel fondo manoscritto della Biblioteca Nazionale Universitaria di Torino ${ }^{1}$ erano presenti, prima dell'incendio sviluppatosi nella notte tra il 25 e il 26 gennaio $1904^{2}$, non meno di 400 manoscritti francesi. Erano collocati per la maggior parte negli scaffali contrassegnati dalle lettere «L» e «M» della sala dei manoscritti, alcuni in banconi della medesima sala, mentre pochi altri erano conservati, con manoscritti in gran parte settecenteschi, nella $1^{\text {a }}$ Sala Riservata. Purtroppo gli scaffali in legno che li contenevano furono aggrediti direttamente dal fuoco, che distrusse una parte dei codici, danneggiando in vario modo quelli superstiti. All'azione del calore si aggiunse quella dell'acqua di spegnimento, utilizzata in grande quantità dai pompieri, la quale fece parzialmente disciogliere gli inchiostri e successivamente favorì l'insorgenza di danni biologici. Ne derivarono, infatti, processi di putrefazione in parte delle pergamene, e proliferazioni di muffe soprattutto nei manoscritti cartacei non adeguatamente prosciugati. Si cercò allora di scongiurare la perdita totale del materiale danneggiato mediante operazioni di disinfezione con soluzioni chimiche, utilizzate in particolare da Icilio Guareschi ${ }^{3}$, professore di chimica nell'Ateneo torinese, o attraverso il prosciugamento ottenuto con cappe aspiranti messe a punto da Piero Giacosa ${ }^{4}$, professore di materia medica nella medesima Università.

Ai gravissimi danni delle componenti materiali dei manoscritti, legature comprese, si aggiunse la distruzione delle indicazioni delle segnature, registrate sulle etichette esterne e sui fogli di guardia, e, in numerosi casi, anche la perdita delle prime carte recanti titoli o altri elementi identificativi. L'identificazione dei codici, per lo più frammentari, fu reso ancor più difficile a causa della distruzione dell'inventario

\footnotetext{
* Ringrazio il prof. Lionello Sozzi per la gentile disponibilità nell'ospitare il presente contributo sulle pagine di «Studi Francesi»; un rigraziamento anche a mia moglie, Lidia De Blasi, per la collaborazione nella revisione del testo.

(1) Per la formazione dei fondi manoscritti della Biblioteca cfr. S. BAssi, Introduzione ai manoscritti della Biblioteca Nazionale, premessa a C. SEGRE MONTEL, I manoscritti miniati della Biblioteca Nazionale di Torino. Volume primo: I manoscritti latini dal VII alla metà del XIII secolo, Torino, Officine Grafiche G. Molfese, 1980, pp. XVII-XXXIII; A. Giaccaria, I fondi medievali della Biblioteca Nazionale Universitaria di Torino. Guida al fondo manoscritto, Alessandria, Edizioni dell'Orso, 1984 (estr. da «Pluteus», 2), pp. 175-194.
}

(2) Notizie dettagliate sui danni e sugli interventi di salvataggio in G. GorRINI, L'incendio della Biblioteca Nazionale di Torino, Torino - Genova, Ed. Renzo Streglio \& C.ia, 1904 (in copertina 1905).

(3) Una puntuale relazione sugli interventi di recupero e di restauro eseguiti nell'Istituto di chimica farmaceutica dell'Università di Torino fu pubblicata da I. GuARESCHI, Osservazioni ed esperienze sul ricupero e sul restauro dei codici danneggiati dall'incendio della Biblioteca Nazionale di Torino, in «Memorie della Reale Accademia delle Scienze di Torino», serie II, LIV (1904), pp. 423-458 e tavv.

(4) Per gli altri interventi di salvataggio e di restauro cfr. P. GIACOSA, Relazione dei lavori intrapresi al Laboratorio di Materia Medica per il recupero e ristauro dei codici appartenenti alla Biblioteca di Torino, in «Atti della R. Accademia delle Scienze di Torino», XXXIX (1903-1904), pp. 3-11. 
topografico del fondo manoscritto, compilato poco dopo la metà dell'Ottocento da Bernardino Peyron 5 e completato negli anni precedenti l'incendio da Carlo Fratí.

Alla reidentificazione dei manoscritti danneggiati si dedicarono alcuni docenti dell'Università di Torino, che meglio conoscevano il fondo manoscritto, con l'attiva collaborazione del personale della Biblioteca ${ }^{7}$.

Rodolfo Renier ${ }^{8}$, professore di storia comparata delle letterature neolatine nell'Ateneo torinese, fu incaricato, in particolare, di identificare i frammenti dei codici italiani e francesi antichi; pubblicò una breve relazione sui risultati di tali lavori, in occasione della recensione al catalogo dei codici italici compilato dal Peyron, ma dato alle stampe soltanto dopo l'incendio'.

Intanto alla direzione della Biblioteca a Francesco Carta ${ }^{10}$, direttore al momento dell'incendio, era subentrato Carlo Frati (1904-1905), ottimo conoscitore del fondo manoscritto, in quanto, come sopra ricordato, aveva provveduto ad aggiornarne l'inventario. Ben presto tuttavia il Frati fu sostituito nelle sue funzioni di direttore da Giuliano Bonazzi ${ }^{11}$ (1905-1909); con la sua lontananza da Torino si fermarono i lavori di identificazione, mentre furono incrementati gli interventi di restauro e di rilegatura di manoscritti.

Intanto le operazioni di recupero, anche per una parte dei manoscritti francesi, continuarono nel Laboratorio restauri della Biblioteca ${ }^{12}$, istituito nel 1904 presso la Facoltà di medicina al Valentino, e sistemato dal 1921 in alcuni locali di via Bogino, nel palazzo del debito pubblico, già destinato fin dal 1907 a diventare la nuova sede della Biblioteca nazionale. Dal 1935 il Laboratorio fu ospitato in due stanze presso il Museo Egizio di Torino, e solo nel 1975 fu definitivamente trasferito nell'attuale sede della Biblioteca Nazionale Universitaria.

(5) Sulla figura e sull'attività del Peyron cfr. D. PEZzI, Bernardino Peyron: commemorazione letta alle classi unite della R. Accademia delle Scienze di Torino nell'adunanza pubblica del di 27 dicembre 1903, Torino, Carlo Clausen, 1904 (estr. da «Atti della R. Accademia delle Scienze di Torino», XXXIX).

(6) Notizie biografiche in M. Parenti, Aggiunte al dizionario bio-bibliografico dei bibliotecari italiani di Carlo Frati, II, Firenze, Sansoni Antiquariato, 1959 , p. 99.

(7) Gaetano De Sanctis, professore di storia antica, aveva provveduto all'identificazione dei manoscritti greci, mentre Carlo Cipolla, professore di storia moderna, in collaborazione con il bibliotecario Carlo Frati, si era occupato dei manoscritti latini: il resoconto sui loro lavori di riconoscimento fu pubblicato, a tre mesi dell'incendio, come Inventario dei codici superstiti greci e latini antichi della Biblioteca Nazionale di Torino, in «Rivista di filologia e d'istruzione classica», XXXII (1904), pp. 385-588. Per il fondo orientale era apparso a cura di I. PIzzi, Il riconoscimento dei Manoscritti arabi, persiani, turchi, ebraici, della Regia Biblioteca Universitaria di Torino, dopo l'incendio del 26 gennaio 1904, in «Atti della R. Accademia delle Scienze di Torino», XXXIX (1903-1904), pp. 1067-1069.

(8) Per notizie biografiche del Renier, nato a Treviso l'11 agosto 1857 e morto a Torino l' 8 gennaio 1915 , vd. le commemorazioni di F. Novati, Rodolfo Renier, in «Giornale storico della letteratura italiana», LXV (1915), fasc. 194-195, pp. 193-198, e di F. Picco, L'operosità scientifica di Rodolfo Renier, in «Nuova antologia di lettere, scienze e arti», CCLIX (1915), pp. 597-604.

(9) R. RenIER, recensione a: Bernardino Peyron - Codices italici manu exarati qui in bibliotheca Taurinensis Athenaei ante diem XXVI Januarii MCMIV asservabantur - Taurini, apud Clausen, 1904 (8 gr., pp. XXXIV-690), in «Giornale storico della letteratura italiana», XLIV (1904), pp. 407-419.

(10) Sulla figura di Francesco Carta cfr. PARENTI, Aggiunte al dizionario..., I, 1957, pp. 232-233; A. Petrucci, Carta, Francesco, in Dizionario biografico degli italiani, XX, Roma, Istituto della Enciclopedia italiana, 1977, pp. 775-776.

(11) Su Giuliano Bonazzi notizie sintetiche in G. DE GREGORI, S. ButTò, Per una storia dei bibliotecari italiani del XX secolo. Dizionario bio-bibliografico 1900-1990, Roma, Associazione italiana biblioteche, 1999, pp. 39-40.

(12) Per gli interventi eseguiti in tempi successivi presso il Laboratorio restauri della Biblioteca cfr. L. TORRI, I restauri dei Codici della Biblioteca Nazionale di Torino, in «MOr EEION», I (1923), pp. 299-306; M. BERSANO BEGEY, Il Laboratorio restauri della Biblioteca Nazionale di Torino, Torino, Accame, 1941 (estr. da «Torino - Rassegna mensile municipale», XXI), pp. 3-6; S. BASSI, Il Laboratorio di restauro della Biblioteca Nazionale Universitaria di Torino, in «Accademie e Biblioteche d'Italia. Bollettino d'informazioni», n.s. II (1962), pp. 79-85. 
Nel 1922, in occasione della pubblicazione dell'inventario dei manoscritti a cura di Francesco Cosentini ${ }^{13}$, il numero dei manoscritti francesi, compresi quelli collocati in altre sezioni, raggiungeva appena le 90 unità: vi furono descritti in maniera sommaria anche i codici in precario stato di conservazione e solo parzialmente consultabili.

A Gino Tamburini ${ }^{14}$, direttore della Biblioteca dal 1932 al 1937, va il merito di aver trascritto le descrizioni di parte dei manoscritti francesi effettuate da Bernardino Peyron, e contenute in un manoscritto di proprietà della famiglia Peyron andato poi disperso. Le trascrizioni, riportate su fogli di recupero, sono consultabili in un volume di fotocopie presso la Sala dei manoscritti e rari delle Biblioteca: esse risultano di particolare interesse, in quanto costituiscono le uniche descrizioni superstiti di numerosi manoscritti francesi, dopo la perdita dell'inventario topografico, già menzionata.

Solo dopo l'ultimo conflitto mondiale fu possibile provvedere alla ricognizione di tutto il fondo manoscritto, compresi i frammenti che per un lungo periodo erano rimasti racchiusi dentro casse in alcuni locali interrati del palazzo dell'Università. In quella circostanza tutti i manoscritti non restaurati, identificati e non, furono sistemati in un locale appositamente attrezzato del terzo piano, dove il bibliotecario Gustavo Vinay $^{15}$ portò a termine l'identificazione di oltre cento manoscritti latini, francesi e italiani. Un riscontro, invece, effettuato sul fondo ebraico in maniera piuttosto affrettata dallo studioso americano Isaiah Sonne fruttò una novantina di identificazioni, talune delle quali errate o incerte.

Negli anni Cinquanta fu possibile aumentare il numero dei restauri grazie alla maggior disponibilità di finanziamenti, che consentirono alla restauratrice Erminia Caudana di essere affiancata nel suo lavoro da un aiutante, il nipote Amerigo Bruna. Si provvide allora allo sbloccaggio di oltre un centinaio di codici membranacei (alcuni dei quali francesi) con i fogli completamente bloccati tra loro, e alla sistemazione delle pergamene debitamente interfogliate in grandi scatole di cartone. Questo primo intervento di restauro, eseguito con maestria, oltre a permettere una ricognizione dei testi contenuti nei singoli manoscritti, ha reso più facile il completamento del restauro stesso $^{16}$.

Dopo il trasferimento della Biblioteca dallo storico palazzo dell'Università in via Po alla sede attuale (1972-73) $)^{17}$, tutti i manoscritti - compresi quelli frammentari non ancora identificati - furono sistemati nell'unico deposito dei manoscritti e dei rari, dove è stato possibile effettuare riscontri anche sul materiale non ancora reidentificato. Numerose identificazioni, infatti, con ritrovamento di manoscritti membranacei e cartacei, più o meno completi, furono effettuate tra il 1974 e il 1977 da Alessandro Vitale-Brovarone, docente dell'Università di Torino ${ }^{18}$.

(13) [Inventario dei manoscritti della Biblioteca Nazionale di Torino, a cura di F. Cosentini], Firenze, Libreria Editrice Leo S. Olschki, 1922 (A. SORBELLI, Inventari dei manoscritti delle Biblioteche d'Italia..., XXVIII).

(14) Un breve profilo biografico in DE GREGORI, ButTò, Per una storia dei bibliotecari italiani..., pp. 168-169.

(15) G. VINAY, Contributo alla identificazione di alcuni manoscritti frammentari della Nazionale di Torino, «Aevum», XXI (1947), 209-232; per la biografia e i suoi studi cfr. G. BRAGA, I. PAGANI, Bibliografia degli scritti di Gustavo Vinay (19121993), in «Studi medievali», $3^{\text {a }}$ serie XL (1999), pp. 395-439.

(16) Biblioteca Nazionale Universitaria di Torino, Manoscritti danneggiati nell'incendio del 1904. Mostra di recuperi e restauri, a cura di A. GIACCARIA, Torino 1986, p. 8.

(17) Cfr. S. BAssi, La Biblioteca Nazionale di Torino. Formazione delle raccolte e sistemazione nella nuova sede, Roma, Nuova Tecnica Grafica, 1975 (estr. da «Associazione Italiana Biblioteche. Bollettino di informazioni», n.s. XV, 1-2), pp. 28-30.

(18) A. Vitale-Brovarone, Verso una ricostruzione del fondo manoscritto della Biblioteca Nazionale di Torino, in «Accademie e biblioteche d'Italia», LI (1983), pp. 458-469. 
Analoghi lavori di reidentificazione furono eseguiti dallo scrivente a più riprese dal 1976, nell'ambito dei suoi compiti relativi alla conservazione e al restauro.

L'identificazione dei testi riportati nei frammenti risulta sovente assai difficile per l'esiguità dei frammenti stessi o per il fatto che i fogli sono tra loro bloccati, come nel caso dei membranacei; l'attribuzione della segnatura precedente l'incendio, poi, è in taluni casi impossibile poiché non tutti i manoscritti sono registrati nei cataloghi conservati, essendo andato distrutto nell'incendio l'inventario topografico di tutti i manoscritti.

Le attribuzioni delle antiche segnature sono state effettuate, pertanto, mediante i riscontri effettuati nei cataloghi e negli strumenti di ricerca sotto elencati, mentre per alcuni manoscritti francesi, in particolare, l'identificazione è avvenuta grazie alle schede presenti in un vecchio catalogo, ordinato per autori o per titoli e conservato attualmente in uno schedario del deposito manoscritti e rari: si tratta nella maggior parte dei casi di schede trascritte a matita da Gino Tamburini.

Le reidentificazioni che di seguito vengono riportate, e quelle relative a manoscritti greci, latini ${ }^{19}$ e italiani ${ }^{20}$, sono state messe a disposizione del gruppo di lavoro dell'Università degli studi di Torino che, coordinato del professor Alessandro VitaleBrovarone, sta provvedendo dal 2001 alla risistemazione dei codici ancora da identificare e alla catalogazione del fondo manoscritto della Biblioteca ${ }^{21}$.

La pubblicazione di questo contributo avviene con ritardo rispetto al compimento dei lavori d'identificazione per una precisa scelta dello scrivente: si è preferito attendere che gli interventi di restauro fossero a buon punto d'esecuzione, proprio per non creare negli studiosi inutili aspettative che diversamente non sarebbe stato possibile soddisfare a causa delle pessime condizioni di conservazione di gran parte del materiale manoscritto. In effetti, oltre ai restauri finanziati in passato dal Ministero della pubblica istruzione e successivamente dal Ministero per i beni culturali e ambientali (attualmente Ministero per i beni e le attività culturali), dal 2000 è in corso l'esecuzione di un progetto straordinario per la risistemazione e il restauro dei manoscritti danneggiati nell'incendio del 1904 e non ancora restaurati ${ }^{22}$.

Le identificazioni per le quali sussista qualche incertezza sono state evidenziate con un punto interrogativo racchiuso in parentesi quadre vicino alla segnatura.

Allo scopo di addivenire ad una ricostruzione ideale, la più completa possibile, del fondo manoscritto originario, sono stati descritti anche due manoscritti ai quali dopo l'incendio era stata attribuita una nuova segnatura perché non erano state reperite descrizioni negli inventari e nei cataloghi superstiti: in questi casi le segnature utilizzate prima dell'incendio sono precedute da un asterisco e seguite, in parentesi tonde, dalle collocazioni attualmente in uso $^{23}$.

(19) A. Giaccaria, Nuove identificazioni di manoscritti greci e latini della Biblioteca Nazionale Universitaria di Torino, in «Aevum», LXXXI (2007), fasc. 2 (in corso di stampa).

(20) Un primo contributo, dedicato a manoscritti d'interesse letterario, è già stato pubblicato: A. GIACCARIA, Identificazione di manoscritti italiani frammentari nella Biblioteca Nazionale di Torino, in «Giornale storico della letteratura italiana», CLXIV, fasc. 526 (1987), pp. 206-218; sono in preparazione per la stampa le descizioni di altre identificazioni relative a circa 170 manoscritti italiani.

(21) A. Vitale-Brovarone, I manoscritti della Biblioteca Nazionale Universitaria: identificazione, riordino, restauro e catalogazione, in «Dall'Universi- tà notizie \& informazioni. Notiziario dell'Università degli studi di Torino», 5 (2002), pp. 16-17.

(22) Cfr. Il patrimonio ritrovato. A cent'anni dall'incendio della Biblioteca Nazionale Universitaria di Torino: la Biblioteca e il progetto di recupero dei fondi manoscritti danneggiati. Introduzione alla mostra (Torino, Biblioteca Nazionale Universitaria, 16 dicembre 2004 - 30 aprile 2005), [Bologna], Trident, 2004, pp. 5-32.

(23) Nel 1904 erano state attribuite nuove collocazioni ai manoscritti recuperati, ma nel 1911 si ritornò alle segnature precedenti l'incendio con l'introduzione delle seguenti sezioni supplementari: $K^{2}$ (per i mss. latini più antichi), $K^{3}$ (per i latini recenti), $L^{2}$ (per i francesi), $L^{3}$ (per gli spagnoli) e 
Si è optato per una descrizione sintetica, riportando (in corsivo) i titoli presenti nei manoscritti stessi o, se non più leggibili, attingendo al catalogo del Pasini, all'inventario del Bencini e ad altre fonti, manoscritte o a stampa. Nei casi in cui non è stato possibile ritrovare il titolo del manoscritto sono stati riportati, in parentesi quadre, i dati relativi al contenuto dello stesso. Alla fine di ogni scheda sono stati indicati in forma abbreviata i riferimenti bibliografici che qui di seguito vengono elencati per esteso con alcune annotazioni esplicative:

- App. al PAsini = Appendice al Pasini, compilata dopo la sistemazione dei fondi manoscritti effettuata, poco oltre la metà dell'Ottocento, da Bernardino Peyron: si tratta d'un indice alfabetico (conservato tra i mss. della Bibliblioteca Nazionale Universitaria), che riporta nell'ambito di ognuna delle sezioni di lingua le indicazioni degli autori, dei titoli sommari e delle segnature;

- BENCINI = Indice de' libri manoscritti ebraici, greci, latini, italiani e francesi i quali la R.M. del Re di Sardegna ha tolti dal suo Regio Archivio per rendere riguardevole la Biblioteca dalla sua Regia Università di Torino... I medesimi sono ordinati... da Francesco Domenico Bencini..., ms. dell'Archivio di Stato di Torino, Regi Archivi, Cat. 9, mazzo $1 \mathrm{n}^{\circ} 1$ (in copia xerografica nella sala manoscritti della Biblioteca Nazionale Univ. di Torino);

- CosENTINI $=[$ Inventario dei manoscritti della Biblioteca Nazionale di Torino, a cura di F. Cosentini], Firenze, Libreria Editrice Leo S. Olschki, 1922 (A. Sorbelli, Inventari dei manoscritti delle Biblioteche d'Italia..., vol. XXVIII);

- GIACOSA = P. GIACOSA, Magistri Salernitani nondum editi: catalogo ragionato della esposizione di storia della medicina aperta in Torino nel 1898, Torino, Fratelli Bocca, 1901;

- Mss. SAluzzo = [Catalogo dei manoscritti già posseduti dal conte Cesare Saluzzo e anteriormente al 1872 passati alla Biblioteca Nazionale di Torino], ms. della Biblioteca Nazionale Univ. di Torino, R.I.10;

- Mostra 1986 = Biblioteca Nazionale Universitaria di Torino, Manoscritti danneggiati nell' incendio del 1904. Mostra di recuperi e restauri, a cura di A. GIACCARIA, Torino 1986;

- PASINI = Codices manuscripti Bibliothecae Regii Taurinensis Athenaei per linguas digesti, et binas in partes distributi, in quarum prima Hebraei, et Graeci, in altera Latini, Italici et Gallici. Recensuerunt, et animadversionibus illustrarunt Josephus Pasinus, Antonius Rivautella, et Franciscus BERTA, Taurini, ex Typographia Regia, 1749: dei manoscritti presenti allora nella Biblioteca furono catalogati quelli ritenuti più importanti, e tralasciati quelli giudicati di scarso interesse; furono distinti per lingue in raggruppamenti effettuati in base ai contenuti e numerati nell'ambito di ciascuna suddivisione per lingua (accanto al numero romano d'ordinamento fu riportata la segnatura introdotta dal Bencini). Le 172 descrizioni di mss. francesi sono contenute nel tomo II, pp. 459-499;

- Peyron-Tamburini = B. Peyron, [Catalogo di codici francesi $]$ (trascrizione a cura di G. Tamburini - direttore della Biblioteca negli anni Trenta - da un manoscritto di proprietà della famiglia Peyron). Si tratta di schede che in parte integrano le descrizioni del Pasini e in parte descrivono mss. pervenuti successivamente alla Biblioteca. In alcuni casi sono ampie e dettagliate, mentre in altri sono assai sommarie. Il manoscritto non è stato ritrovato nel Fondo Peyron acquisito nel 1970 dalla Biblioteca.

- VINAY = G. VINAY, Contributo alla identificazione di alcuni manoscritti frammentari della Nazionale di Torino, in «Aevum», XXI (1947), pp. 209-232;

- Vitale-Brovarone = A. Vitale-Brovarone, Verso una ricostruzione del fondo manoscritto della Biblioteca Nazionale di Torino, in «Accademie e biblioteche d'Italia», LI (1983), pp. 458-469;

- WAHLGREN = E. G. WAHLGREN, Renseignements sur quelques manuscrits français de la Bibliothèque Nationale di Turin, Uppsala, Almquist \& Wiksell Boktryckeri-A.-B., 1934 (estr. da «Studier i Modern Spraokvetenskap utg. av Nyfilologiska Sâllskapet i Stockholm», XII).

$Q^{2}$ (per gli italiani), nelle quali furono collocati i mss. conservati prima dell'incendio nella $1^{\mathrm{a}}$ Sala
Riservata e quelli che comunque erano sprovvisti della vecchia segnatura. 
L.III.4

L.III.6

L.III.23

L.III.27

L.IV.4

L.IV.5
Les coups d'hazard. Comedie traduite de l'Italien par Jean Louvart, advocat en Parlement et interprete es langues pour le Service de S.A.R. le Duc de Savoye.

Cart., sec. XVII, mm 300 x 215, ff. 38. Bruciato sui dorsi e sui margini in testa (con lieve perdita di testo), andò smembrato e scompaginato; la parte reperita è quella finale, utilizzabile in parte. Pasini Gall. no 124 (l.V.35); Peyron-Tamburini (L.III.4)

Le livre des philosophes translate de latin en françois par Misser Guillaume de Tignonville; Livre de la moralité des nobles hommes et des gens du peuple fait sur le gieu des eschez [de Jacques de Cessoles] translate de latin en françois par Frere Jehan de Vignay.

Membr., sec. XIV, mm 130 x 95, ff. 2 frammentari (solo parzialmente leggibili a causa dei gravi danni derivati dal fuoco e dall'acqua di spegnimento) da unire a quelli identificati e restaurati in passato. PAsini Gall. nº 59 (i.VI.27); Cosentini n 1648

Jerosme Justinian (Girolamo Giustiniani), Histoire de l'Isle de Chio et autres Isles et villes de la Grece.

Cart., sec. XVII, mm 165/260 x 210, ff. 3 frammentari (da unire alle parti identificate da Vitale-Brovarone), notevolmente danneggiati dal fuoco e leggibili in parte.

Bencini f. 627v (L.III.16); PASini Gall. nº 110 (l.III.16); WAHLGREN p. 32

Histoire de Savoye [contenente soltanto il proemio e la vita di Beroldo].

Cart., sec. XVII, mm 280/295 x 180/200, ff. 128, I. Danneggiato parzialmente dal fuoco nella parte superiore. Restaurato a fogli sciolti; utilizzabile in gran parte.

Pasini Gall. no 113 (l.III.23); Peyron-Tamburini (L.III.27)

[Recueil des conférences tenues à Tolède, dans le palais de l'empereur, entre l'archevesque d'Embrun et M. De Selva, premier président de Paris, ambassadeur de la régente de France, d'une part, et de l'autre, le vice-roi de Naples, le chancelier et le grand-maître de Charles-Quint, au sujet de la délivrance de François $\left.I^{e}\right]$.

Cart., sec. XVI, mm 200/290 x 150/190, ff. 112 frammentari. Gravemente danneggiato dal fuoco che distrusse le zone marginali con perdita di testo; restaurato a ff. sciolti. Testo parzialmente leggibile.

Bencini f. 632v (L.II.34); PASini Gall. nº 101 (l.II.34); WAHLGRen p. 11

[Ms. miscellaneo contenente tra l'altro:] Le livre du roy Ponthus; ... Gauthier de Metz, L'image du monde.

Cart., sec. XV, mm 255 x 110, ff. 58 frammentari (parte dell'ultimo testo contenuto nel ms., parzialmente leggibile), restaurati recen- 
temente: da unire alla parte identificata subito dopo l'incendio e a quella reperita da Vitale-Brovarone.

PASINI Gall. n 12 (g.I.2); Cosentini n ${ }^{\circ}$ 1668; WAHLGREN pp. 21-22

L.IV.12 Martin Le Franc, L'estrif de vertu et fortune divisé en troys livre.

Cart., sec. XV, mm 145 x 155/160, ff. 3 frammentari (di cui uno iniziale, e gli altri due numerati 150 e 151 nella cartulazione settecentesca) da aggiungere alla parte identificata subito dopo l'incendio e a quella reperita successivamente da Vinay. Leggibili solo in parte. PASINI Gall. n 50 (i.IV.32); Cosentini n ${ }^{\circ} 1672$; VinaY n 88

L.IV.17 [Miscellanea di trattati di medicina e chirurgia, tradotti dal latino, tra i quali:] Henry de Mondeville, Anathomie, Cirurgie; Nicholas, Antidotaire; Platearius, Circa instans.

Cart., sec. XV, mm 150/220 x 90/185, ff. 31 frammentari, con testo solo parzialmente utilizzabile, da aggiungere alle parti identificate da Vinay e successivamente restaurate.

Pasini Gall. n 78 (k.V.28); Wahlgren p. 31; VINAY n 90

L.IV.18 [Chronique dès l'origine du monde jusqu'au $\mathrm{XV}^{\mathrm{e}}$ siècle].

Cart., sec. XV, mm 140 × 100 circa, ff. 52 frammentari (superstiti le porzioni al piede, parzialmente corrose in corrispondenza delle scritture): di minima utilità a causa della limitata conservazione di testo.

BenCini f. 622r (L.II.25); PASIni Gall. nº 96 (l.II.25); WAHLGREN p. 31

L.IV.24 [Poème prolixe anonyme sur les faits du Christ et de sa famille]. Cart., sec. XV, mm 195/220 x 95/150 , ff. 2 frammentari (uno della parte iniziale, l'altro di quella finale del ms.), utilizzabili solo parzialmente a causa dei rilevanti danni del fuoco e dell'acqua; da aggiungere alla parte identificata subito dopo l'incendio, successivamente restaurata e rilegata in volume, e a quella reperita da Vitale-Brovarone.

Pasini Gall. n 121 (l.V.31); Cosentini n 1676 (con titoli non pertinenti); WAHLGREN p. 45

L.IV.26 Jehan Froissart, Chroniques (troisième livre).

Cart., sec. XV, mm 210/270 x 120/130, ff. 222 frammentari, cui devono essere aggiunti $146 \mathrm{ff}$. reperiti dal Vinay e ritenuti (con qualche dubbio) come appartenenti al ms. L.V.7, successivamente restaurati. Testo utilizzabile solo in parte limitata a causa dei gravi danni derivati dal fuoco e dall'acqua di spegnimento. Conferma del contenuto in un'annotazione ms. autografa di Costanzo Gazzera, prefetto della Biblioteca tra il 1844 e il 1859, riportata nel catalogo del Pasini in uso nella sala manoscritti della Biblioteca.

BENCINI f. 623r (L.II.32); PASINI Gall. nº 100 (l.II.32); VINAY n 94 (L.V.7)

L.IV.27 Les fleurs des chroniques (Vies des Papes jusqu'à Jean XXII, par Frère Bernard Guy; Vies des Empereurs Romains et Germaniques jusqu'à Henri, comte de Luxembourg; Vies des Rois de France jusqu'à Philippe VI). 
Cart., sec. XV, mm 210/260 x 115/135, ff. 74 frammentari (in origine 282), restaurati sciolti: utilizzabili in minima parte a causa degli estesi danni causati del fuoco.

Pasini Gall. n 107 (l.III.7); Peyron-Tamburini (L.IV.27); WahlGREN p. 32

L.IV.32 La vie du roy Ponthus.

Cart., sec. XIV-XV, mm 162 x 120, 1 f. frammentario, con testo solo parzialmente leggibile, a causa dei danni del fuoco; da unire alla parte identificata in passato da Vinay.

Pasini Gall. n 11 (g.I.1); WAhlgREN p. 29; VinaY n 91

L.V.6 Thomas III de Saluces, Le Chevalier errant.

Membr., sec. XV, mm 70/115 x 60/80, ff. 14 frammentari, da aggiungere alle pergamene identificate subito dopo l'incendio e sottoposte in passato ad interventi di restauro. Utilizzabili in minima parte dopo il restauro, a causa dei gravissimi danni derivati dal fuoco e dall'acqua di spegnimento.

PAsini Gall. n 25 (g.I.39); Cosentini n 1680; Mostra 1986 n 18

L.V.7 [Traité et deux chroniques sur les guerres des Anglais en France].

Cart., sec. XV, mm 70/135 x 85/165, ff. 170 frammentari, a causa dei gravissimi danni del fuoco che li distrusse nella parte superiore: testo leggibile in minima parte. I $146 \mathrm{ff}$. identificati da Vinay come parte del ms. L.V.7 in realtà appartengono al ms. L.IV.26, con il quale devono essere riuniti.

PAsini Gall. n 103 (l.II.36); Wahlgren p. 11; Vinay nº 94 (L.V.7)

L.V.10 Livre de la moralité des nobles hommes fait sur le jeu des eschecs [de Jacques de Cessoles] traduit par Jehan de Vignay.

Membr., sec. XIV-XV, mm 190 x 125, ff. 2 (39 e 40 nella cartulazione settecentesca), da unire alla restante parte del ms. identificata dopo l'incendio e successivamente restaurata con numerazione parzialmente scorretta.

PAsini Gall. n 114 (l.IV.29); Cosentini n 1682; WAHLgren p. 46

L.V.30 Le Romans de Lancellot.

Cart., sec. XV, mm 130/180 x 80/110, ff. 51 frammentari, da unire alla parte identificata e restaurata in passato; utilizzabili solo in parte a causa dei gravi danni derivati dal fuoco e dall'acqua di spegnimento.

PAsini Gall. n 132 (g.I.12); Cosentini n 1688

L.VI.1-3 Abrégé des mémoires des Messires Martin et Guillaume du Bellai seigneurs de Langei contenant les choses les plus remarquables arrivées pendant les dernières années de Louis XII et sous le règne de François I, rois de France, divisé en dix livres avec le sommaire à la tete de chaque livre [Tomes I-III].

Cart., sec. XVIII, mm 130/224 x 120/185, Tome I: ff. 335+ 6 bianchi; Tome II: ff. 147 frammentari + 3 bianchi;. Tome III: ff. 236 in parte frammentari. I tre tomi subirono danni dal fuoco in maniera differenziata: meno gravi nel primo, dove il testo è leggibile in gran parte, 
molto più gravi negli altri due (in particolare nel secondo dove manca la prima metà dei ff. e quelli conservati presentano rilevanti perdite di testo). Restaurati recentemente a ff. sciolti; utilizzabili solo in parte. Identificazione basata sul vecchio catalogo a schede dei mss. s.v. Abregé

L.VI.5 Mémoire cencernant la Province de Languedoc dressé par Monsieur de Basville, Conseiller d'Etat et Intendant en Languedoc en 1698.

Cart., sec. XVII ex., mm 220 x 170, ff. 205, I. Danneggiato dal fuoco sulle zone marginali (particolarmente al piede nei primi $25 \mathrm{ff}$. con parziale distruzione di testo), ma quasi completamente leggibile, restaurato a ff. sciolti.

Identificazione basata sul vecchio catalogo a schede dei mss. s.v. Mémoire

L.VI.6 César Brancadoro, Meditations sur les Tombeaux de Louis XVI, Roi de France, de Marie-Antoinette son auguste Epouse et de Madame Elisabeth de France, par Monseigneur César, des Comtes de Brancadoro, Archéveque de Nicibe, ci-devant Nonce apostolique à Bruxelles et actuélement Sécrétaire de la Sacré Congrégation de propaganda fide.

Cart., sec. XVIII, mm 218 x 175, ff. II, 194 (di cui 4 non scritti all'inizio e 2 alla fine). Parzialmente danneggiato dal fuoco e dall'acqua di spegnimento; restaurato a ff. sciolti, con testo quasi completamente leggibile.

Identificazione basata sul vecchio catalogo a schede dei mss. s.v. Brancadoro

L.VI.7 Abrégé de l'bistoire de Savoye.

Cart., sec. XVIII, mm 215 x 150, ff. 70, di cui alcuni frammentari. Danneggiato dal fuoco sulle zone marginali con parziale interessamento del testo. Restaurato a ff. sciolti, leggibili in buona parte.

Identificazione basata sul vecchio catalogo a schede dei mss. s.v. Abrégé

L.VI.9 Traitté sommaire de l'artillerie party in trois livres [presente solo il primo libro].

Cart., sec. XVI, mm 215 x 145, ff. I, 14, I. Danneggiato dal fuoco nelle zone perimetrali e dall'acqua di spegnimento (ff. in parte carbonizzati e macchiati dagli inchiostri parzialmente disciolti); utilizzabile in gran parte.

Identificazione basata sul vecchio catalogo a schede dei mss. s.v.Traitté

L.VI.12 [?] Abrégé historique de la Maison de Savoye.

Cart., sec. XVIII, mm 210 x 155, ff. 85 frammentari (il primo e l'ultimo non scritti). Danneggiato perimetralmente dal fuoco (in maniera più accentuata al piede con interessamento di parte del testo); leggibile in parte. L'opera prima dell'incendio era costituita da tre volumetti (rispettivamente I, II, IV), di cui si è reperito solo l'ultimo.

Identificazione basata sul vecchio catalogo a schede dei mss. s.v. Abrégé (L.VI.10-12) 
L.VI.14 [Miscellanea di astrologia e alchimia, in francese e in latino, parzialmente in versi].

Cart., sec. XV, mm 195 x 130, ff. 40 (da unire alla prima parte del ms., identificata da Vinay), con alcuni disegni colorati. Danneggiato dal fuoco che lo bruciò marginalmente (con parziale distruzione di testo), e dall'acqua di spegnimento, andò scompaginato. Restaurato in tempi successivi a ff. sciolti, leggibili in gran parte.

BenCini f. 485r (J.I.28); Peyron-TAMburini (L.VI.14); Vinay nº 96; Mostra $1986 n^{\circ} 62$

L.VI.18 Des systemes des autheurs qui ont traitté de fortification.

Cart., sec. XVIII, mm 210x 145, ff. 81 frammentari (in origine 262), con numerosi disegni di fortificazioni. Bruciato in profondità al piede con perdita di parti scritte, andò scompaginato; la parte reperita è solo parzialmente utilizzabile.

Identificazione basata sul vecchio catalogo a schede dei mss. s.v. Des systemes

M.I.2-6/7 Mémoires sur les actions les plus eclatantes et sur les droits les plus pretieux des Comtes et Ducs chefs de la Roiale Maison de Savoie.

Cart., sec. XVIII in., mm 425 x 275/280, 5 v.: tomo I: ff. 147 (comprende la storia da Beroldo ad Amedeo VII); tomo II: ff. 79 (contiene la storia da Amedeo VIII a Carlo II); tomo III: ff. 87 (contiene la storia da Filippo II settimo Duca a Emanuele Filiberto); tomo IV: ff. 73 (contiene la vita di Carlo Emanuele I); tomo V: ff. 76 (contiene la vita di Vittorio Amedeo II, ed è registrato con la segnatura M.I.6/ 7). Restaurati e rilegati in volumi, essendo completi e ordinati, con testo completamente utilizzabile.

Peyron-Tamburini (M.I.2-6/7)

M.I.9 Mémoires et négociations secrètes de la Cour de Turin, contenant les Rélations que $M^{r}$ De Phelippeaux ambassadeur de France à Turin a écrites au Roi son maître depuis le 25 octobre 1703 jusqu'au temps de son échange le 21 may 1704, avec une lettre de S. M. T. C. et autres mémoires au sujet de la présente guerre d'Italie.

Cart., sec. XIX in., mm 290 x 230, ff. 45 frammentari (di cui 3 non scritti in fine). Bruciato sul dorso e sui margini con parziale perdita di testo, andò scompaginato; restaurato a ff. sciolti leggibili solo in parte a causa dei gravi danni subiti.

App. al Pasini f. 236v s.v. Phelippeaux; Peyron-Tamburini (M.I.9)

M.I.18-19 Mémoires de Monsieur de Saintot introducteur des ambassadeurs. Cart., sec. XVIII in., mm 185/245 x 235/240, ff. 165 frammentari di cui 2 bianchi (in origine vol. I: pp. 397; vol. II: pp. 376). I due mss. danneggiati gravemente dal fuoco con notevole perdita di testo (nella metà superiore) e dall'acqua, andarono scompaginati e dispersi in mezzo ai frammenti da identificare; restaurati a ff. sciolti, sono utilizzabili in parte limitata.

Peyron-Tamburini (M.I.18-19)

M.II.1

Traitté de la nouvelle pratique de la Royalle fortiffication divisé en deux 
partyes, dans la premiére des quelles sera parlé du moyen de construire en deux manieres les neuf figures Regulieres et avec le simple Regle et le Compas comme aussy de la Juste mesure et dimmension tant de leurs lignes comme de la qualité de leurs angles. Dans la seconde Partye seront resouls tous les doubtes qui concernent les Poligones Irregulieres par la cognoissance que l'on aura des partyes Essentielles de la Reguliere et Royalle fortification, le tout en faveur du nouveau soldat.

Cart., sec. XVII, mm 215/300 x 170/190, ff. 82 frammentari. Bruciato in profondità sui dorsi e sui margini superiori, subì ulteriori danni dall'acqua di spegnimento che fece sbiadire le scritture; utilizzabile solo parzialmente.

App. al Pasini f. 230v s.v. Fortificazione; Peyron-Tamburini (M.II.1)

M.II.2 Livre des douze portes de George Riplee; Abbregé d'Alchimie, ou comentaire sur la Recapitulation des 12 portes de George Riplee par Irenee Philalete; Traitté du Mercure et de la Pierre des philosophes de George Riplee; La vision de George Riplee expliquee par Irenee Philalete; Epistre de George Riplee à Edouard IV Roy d'Angleterre expliquee par Irenee Philalete; La Mouelle d'alchimie, seconde partie de livre premier de George Riplee expliquee par Irenee Philalete; Description de Mercure de philosophes.

Cart., sec. XVIII in., mm 335 x 205, ff. I, 195 (bianchi 2 di quelli iniziali). Bruciato perimetralmente dal fuoco senza danni per il testo; restaurato a ff. sciolti conservati in cartella: completamente leggibile.

Peyron-TAmburini (M.II.2)

M.II.3 Abregé du grand oeuvre des Philosophes tiré du manuscrit de Josias, qui est le vray Lion rouge; De medicina universali, quae ex nigra venenosa et volatili magnesia saturnina preparatur; Oeuvre donnée par Monsieur de Vaudremont de Cologne..., c'est l'oeuvre du feu de l'antimoin d'Hongrie; Le Testament d'or; Lettre de l'apothicaire de village au Procureur de la Communeauté des apothicaires d'Angers au sujet du Procès d'entr' eux et le Corps des Marchands pour leur marche à la procession du Sacre et l'explication de la lettre.

Cart., sec. XVIII in., mm 345 x 220, f. 1 (p. 41, ultima del secondo trattato) e ff. 107 da unire alla parte presente al posto. Il ms. bruciato sulle zone marginali, andò scompaginato: i primi due trattati reperiti in un passato furono sottoposti a restauro e rilegati in volume (nell'ordine inverso rispetto a quello documentato nella descrizione sotto menzionata); la parte mancante è stata identificata recentemente e fatta restaurare a ff. sciolti, conservati in cartella.

Peyron-Tamburini (M.II.3)

M.II.4 Traité abregé des règles plus essentielles pour le lavis ou Dessein en plan dont se doivent servir ceux qui professent la science d'ingenieur par Ricaud.

Cart., sec. XVIII, mm 360 x 210, ff. 5 (frontespizio, con disegni a colori, e parte iniziale del ms. originariamente di pp. 24, andato smembrato durante l'incendio). Testo utilizzabile in parte.

PeYron-TAmburini (M.II.4) 
M.II.6 [Conte Perrone di S. Martino], Essai sur le commerce de nôtre Etat. Cart., sec. XVIII, mm 305 x 210, ff. II, 176. Bruciato al piede con parziale perdita di testo; restaurato a fogli sciolti, utilizzabile in discreta parte.

Peyron-Tamburini (M.II.6)

M.II.7 Relation de la campagne de 1746 faite en Italie par les armées combinées d'Espagne, de France, de Naples et Gênes contre les troupes Imperiales et celles de Roy.

Cart., sec. XVIII, mm 310 x 220, ff. 81 frammentari. Bruciato in profondità nella metà inferiore con rilevante perdita di testo; restaurato a ff. sciolti, leggibili solo in parte.

Peyron-TAmburini (M.II.7)

M.II.10 Mémoires pour servir à l'bistoire des Albigeois.

Cart., sec. XVIII, mm 310/340 x 190/225, ff. 107 frammentari. Bruciato al piede con parziali danni al testo; restaurato a ff. sciolti; utilizzabile solo parzialmente.

Peyron-Tamburini (M.II.10)

*M.II.12

(ora L'.I.1)

Architecture de Vitruve traduite en françois par Jean Martin, avec figures.

Cart., sec. XVI ex., mm 352 x 235, ff. III, 86 + 1 tav. doppia silografica in fondo, illustrato con numerosi disegni ad inchiostro. $\mathrm{Al} \mathrm{ms}$. parzialmente danneggiato dal fuoco e dall'acqua fu attribuita, dopo I'incendio, la nuova segnatura L2.I.1, non essendo stato reperito negli inventari e nei cataloghi allora noti. Restaurato alcuni anni or sono a ff. sciolti conservati in cartella; completamente utilizzabile.

Peyron-Tamburini (M.II.12)

M.II.13 Isaac Martin, De la fortiffication par M. Isaac Martin Secretaire du Roy. Cart., sec. XVI-XVII, mm 290 x 240, ff. 60 con disegni a penna (uniti in occasione del restauro ai 10 iniziali, identificati da Vitale-Brovarone). Bruciato perimetralmente (in profondità con perdita di testo al piede), andò scompaginato; in fase di recupero subì varie lacerazioni con asportazione nei margini superiori. Utilizzabile in parte. App. al Pasini f. 234v s.v. Martin; PeYron-Tamburini (M.II.13)

M.II.15 Mémoires de la Regence de Marie Jeanne Baptiste de Nemours duchesse mère de Savoye et de la continuation de son gouvernement depuis la majorité du Duc son fils jusqu'à son mariage.

Cart., sec. XVIII-XIX, mm 310 x 230, ff. 109 di cui 1 bianco. Bruciato al piede con interessamento dello scritto, andò scompaginato con dispersione di una notevole parte dei ff. (utilizzabili parzialmente quelli reperiti).

App. al Pasini f. 235r s.v. Mémoires; Peyron-Tamburini (M.II.15)

M.II.17 Mémoires bistoriques sur la negociation de la France et de l'Angleterre dépuis le 26 mars jusques au 20 septembre 1761.

Cart., sec. XVIII, mm 340 x 242, ff. 113. Parzialmente danneggiato dal fuoco sui dorsi e sui margini; restaurato a fascicoli sciolti conservati in apposita cartella; quasi completamente utilizzabile. 
Peyron-Tamburini (M.II.17)

M.II.18 Lettres et memoires de Monsieur le Cardinal Mazarin à Messieurs Le Tellier et De Lyonne contenans le secret de la negociation de la paix des Pyrenées dans conférences tenues à S. Jean De Luz entre le dit sieur Cardinal et Dom Louis D'Haro.

Cart., sec. XVII, mm 330 × 225, ff. I, 405. Parzialmente bruciato nelle zone marginali, senza distruzione di testo (completamente leggibile); restaurato a ff. sciolti, conservati in apposita cartella.

PEYRON-TAMBURINI (M.II.18)

M.II.20 Recherches des titres qui ont aquis à la Royale Maison de Savoye les états qu'elle possede.

Cart., sec. XVIII, mm 330 x 228, ff. 71 (l'ultimo non scritto). Lievemente danneggiato dal fuoco sui bordi; restaurato e rilegato in volume, essendo risultato completo e in ordine.

Mss. Saluzzo f. 35r; Peyron-Tamburini (M.II.20)

M.II.22 [Andrea Provana di Leyni], Relation du siège de Vienne, faite par un officier de la famille Provana de Leyni qui prit part à la défense.

Cart., sec. XVIII, mm 340 x 225, ff. 59 (bianco il primo). Lievemente bruciato sui bordi, restaurato a ff. sciolti; testo quasi completamente leggibile.

PEYRON-TAMburini (M.II.22)

M.II.23 Reglement pour le Regiment aux Gardes à pied de la Couronne de Pologne, 1774.

Cart., sec. XVIII, mm 332 x 230, ff. 77. Bruciato sui dorsi e lievemente sui margini; restaurato a ff. sciolti, utilizzabile quasi completamente.

App. al Pasini f. 238v s.v. Reglement; Peyron-TAmburini (M.II.23)

M.II.24 Detail de tous les faits remarquables de la dernière guerre d'Italie commencée en 1701 et finie en 1712 avec des remarques sur les opérations.

Cart., sec. XVIII, mm 330 × 220, ff. 78 (di cui 7 bianchi in fine) uniti, in occasione del restauro, ai 2 iniziali identificati da Vitale-Brovarone. Bruciato lievemente sulle zone marginali; restaurato a ff. sciolti, completamente leggibili. Il ms. con il medesimo titolo e contenuto, identificato dopo l'incendio con la doppia collocazione M.II.24-25, in realtà è il ms. M.II.25.

App. al Pasini f. 227v s.v. Detail; Peyron-Tamburini (M.II.24-25)

M.II.26 Jean Tonso, La vie d'Emanuel Philibert Duc de Savoye Ecrite par Jean Tonso, Juresconsulte Milanois mise en François.

Cart., sec. XVII, mm 320 x 216, ff. 314 (bianchi il primo e gli ultimi 3). Lievemente danneggiato dal fuoco e più gravemente dall'acqua di spegnimento; restaurato a ff. sciolti conservati in cartella, quasi completamente utilizzabile.

PEYRON-TAMburini (M.II.26) 
M.II.28 Leçons de calcul integral de M. De Cauchy à l'Ecole Royale Polytechnique, 1821-22, $2^{\text {ème }}$ année [recueillies par] Villatte.

Cart., sec. XIX in. mm 315 x 220, ff. 100 (bianchi il primo e gli ultimi 18). Bruciato sui margini nella parte superiore, senza danni al testo; completamente utilizzabile. Restaurato a ff. sciolti. Mss. Saluzzo f. 7r; Peyron-Tamburini (M.II. 28)

M.II.30 Du vicariat de l'Empire. Composé en 1762 par M. de [Montagnini]; Mémoire dressé par le Procureur général de S M. Baron Favrat de Bellevaux sur le vicariat général et perpetuel qui appartient à la Rö̈ale Maison dans les Etats de sa Domination et sur le vicariat, qu'elle a droit d'exercer en Italie dans le tems d'interrêgne et de troubles.

Cart., sec. XVIII, mm 330/340 x 225, ff. 176 di cui 2 bianchi in fine. Bruciato sui margini nella parte superiore, con limitati danni al testo, andò scompaginato: la prima parte era conservata in mezzo ai frammenti da identificare, mentre la seconda era stata collocata con il materiale identificato, ma con indicazioni di segnatura non pertinente (M.V.10 o 14). Restaurato a ff. sciolti, quasi del tutto leggibili.

Mss. Saluzzo f. 16r e 29r (M.II.20 corretto in 30); App. al PasinI f. 230 r s.v. Vicariat (con segnatura M.II.20); PEYRON-TAMBurini (M.II.30)

M.II.31 Essai d'une relation de l'etat présent de la Cour de Vienne. A Turin le 28 octobre 1749.

Cart., sec. XVIII, mm 330 x 210, ff. III, 63 (bianchi gli ultimi 5). Bruciato sui bordi senza danni al testo, completamente utilizzabile; restaurato a ff. sciolti.

Peyron-TAmburini (M.II.31)

M.II.32 Relation de la campagne de 1746 faite en Italie par les armées combinées d'Espagne, de France, de Naples et Génés contre les troupes Imperiales et celles de Roi.

Cart., sec. XVIII, mm 325 x 210, ff. I, 97 (bianco il primo). Bruciato superficialmente sui dorsi e sui margini in testa con parziali danni al testo; restaurato a ff. sciolti, utilizzabili in parte.

Peyron-Tamburini (M.II.32)

M.II.33 Almanach pour l'an MDLXXIX. Avec les tablettes pour ecrire sur chacun jour...

Cart., sec. XVI, mm 315 x 195, ff. 46 (bianchi i primi 2 e gli ultimi 5). Danneggiato dal fuoco sui dorsi e sui margini (fino al testo nella parte superiore); restaurato a ff. sciolti, conservati in cartella, utlizzabili quasi completamente.

Bencini f. 293r (J.II.15); Peyron-Tamburini (M.II.33-34)

M.II.34 Almanach pour l'an MDLXXIX. Calculé sur le meridien de Savoye, Piemont et lieux circonvoisins.

Cart., sec. XVI, mm 310 x 200, ff. 44 (bianco il primo e gli ultimi 3). Bruciato parzialmente sulle zone marginali in testa; restaurato a ff. sciolti, conservati in cartella, utilizzabili quasi del tutto.

Bencini f. 293 r (J.II.15); Peyron-TAmburini (M.II.33-34) 
M.II.35 Recueil des sentimens de l'Assemblée militare qui se tient chez S. A. Monseigneur le Marquis de Suse, MDCCXIV.

Cart., sec. XVIII, mm 290 x 210, ff. 82 (bianchi gli ultimi 3); scritture rosse e blu sbiadite. Danneggiato dal fuoco e dall'acqua di spegnimento, con parziale perdita di testo nella parte superiore; restaurato a ff. sciolti, leggibili in gran parte.

Peyron-TAmburini (M.II.35)

M.II.36-37 Bonnaventure Dargonne, Histoire de la théologie dans tous les siècles pour servir de preuve à la perpetuité de la tradition contre les béretiques et les autres ennemis de l'Eglise. Ouvrage posthume du venerable Père Dom Bonnaventure Dargonne Charteux. Tome I: La théologie des anciens Patriarches depuis Adam... jusqu'aux premiers Pères de l'Eglise; ... Tome II: La théologie des Pères de l'Eglise.

Cart., sec. XVIII, tomo I: mm 280/320 x 200/215, ff. 331; tomo II: mm 270/305 x 180/215, ff. 271. I due manoscritti subirono danni dal fuoco sulle zone marginali con parziale perdita di testo (utilizzabile solo in parte); sono stati restaurati a ff. sciolti, conservati in apposite cartelle.

Peyron-Tamburini (M.II.36-37)

M.II.38 Mémoires de la Regence de Marie Jeanne-Baptiste de Savoye, duchesse mère de Savoye et de la continuation de son gouvernement depuis la majorité du Duc son fils jusques à son mariage.

Cart., sec. XVII, mm 270/320 x 170/200, ff. 236 in parte frammentari. Bruciato sui dorsi e sui margini con rilevanti danni, aggravati dalle infiltrazioni d'acqua non prosciugate; restaurato a ff. sciolti, leggibili in parte.

PEYRON-TAMBurini (M.II.38)

M.II.39 [Poesie anonime dedicate al Duca di Savoia].

Cart., sec. XVI, mm 285 x 180, ff. 30 (bianchi i primi 3). Bruciato in profondità sui margini con perdita di testo (leggibile solo in parte). Restaurato a ff. sciolti.

PeYron-TAMburini (M.II.39)

M.II.40 Relation de la campagne du 1745 faite en Italie par [les armées Espagnole,] Françoise, Napolitaine et Genoise contre l'armée [Autrichienne et] celle du Roi.

Cart, sec, XVIII, mm 325 x 205, ff. 49. Bruciato perimetralmente con parziale distruzione del testo, leggibile solo in parte; restaurato a ff. sciolti.

Peyron-TAmburini (M.II.40)

M.II.41-42 Ceremonial de la Cour de France tiré des mémoires de monsieur de Saintot.

Cart, sec, XVIII, mm 320 x 210, ff. $150+4$ bianchi (in origine vol. I: pp. 191 + indice; vol. II: pp. 118). Danneggiati notevolmente dal fuoco e dall'acqua, con parziale distruzione di testo, andarono scompaginati in mezzo ai frammenti da identificare. Restaurati a ff. sciolti, sono utilizzabili solo in parte. 
Mss. Saluzzo f. 40r; App. al Pasini f. 240v s.v. Sainctot; PeyronTAMBURINI (M.II.41-42)

M.III.1 Recueil de lettres tirées des Registres des Roiales Archives depuis l'année 1649 jusques en 1668.

Cart, sec. XVIII, mm 260 x 190, ff. 168 in parte frammentari. Bruciato perimetralmente, subì ulteriori danni dall'acqua di spegnimento; restaurato a ff. sciolti leggibili in parte.

PEYROn-TAmburini (M.III.1-9)

M.III.3 [Recueil de lettres tirées des Registres des Roiales Archives]-Registre 1720-23.

Cart, sec. XVIII, mm 290 x 198, ff. 125 in parte frammentari. Danneggiato sui dorsi e sui margini dal fuoco; restaurato a ff. sciolti utilizzabili solo in parte.

Peyron-TAmburini (M.III.1-9)

M.III.4 [Recueil de lettres tirées des Registres des Roiales Archives] - Registre 1723-24.

Cart, sec, XVIII, mm 290 x 198, ff. 95 in parte frammentari. Danneggiato del fuoco e dell'acqua, con parziale perdita di testo; restaurato a ff. sciolti, utilizzabili solo in parte.

Peyron-Tamburini (M.III.1-9)

M.III.5 [Recueil de lettres tirées des Registres des Roiales Archives] - Registre des lettres 1724-1728 à Cambray et à Paris.

Cart, sec. XVIII, mm 290/330 x 190/215, ff. 126 in parte frammentari. Bruciato sulle zone marginali con parziale distruzione di testo; restaurato a ff. sciolti, utilizzabili in parte.

PEYRON-TAMburini (M.III.1-9)

M.III.6 [Recueil de lettres tirées des Registres des Roiales Archives] - Registre 1724-27.

Cart, sec. XVIII, mm 308/322 x 180/200, ff. 126 in parte frammentari (gli ultimi 2 non scritti). Danneggiato per l'azione del fuoco e dell'acqua di spegnimento (che fece disciogliere parzialmente gli inchiostri causando ampie macchie); restaurato a ff. sciolti con testo leggibile in gran parte.

Peyron-Tamburini (M.III.1-9)

M.III.7 [Recueil de lettres tirées des Registres des Roiales Archives] - Registre des lettres depuis avril 1727.

Cart, sec. XVIII, mm 300 x 180, ff. 80 (di cui 1 bianco). Bruciato sulle parti marginali, con ulteriori danni per l'acqua in parte dei $\mathrm{ff}$., andò scompaginato; restaurato a ff. sciolti (14 ff., notevolmente danneggiati dalle infiltrazioni d'acqua sono ancora da restaurare), utilizzabili solo in parte.

Peyron-Tamburini (M.III.1-9)

M.III.8 [Recueil de lettres tirées des Registres des Roiales Archives] - Registre des lettres de la Cour commencé le 10 octobre 1746 et fini le 20 juillet 1748. 
Cart, sec, XVIII, mm 314 x 200, ff. 193 in parte frammentari. Danneggiato per l'azione del fuoco (con parziale distruzione di testo); restaurato a ff. sciolti; solo parzialmente leggibili. Peyron-Tamburini (M.III.1-9)

M.III.9 [Recueil de lettres tirées des Registres des Roiales Archives]-Registre des lettres de la Cour commencé le 28 juillet 1748 à Savone.

Cart, sec. XVIII, mm 295 x 185, ff. 115 in parte frammentari. Danneggiato dal fuoco, andò in parte disperso (ancora mancante la parte iniziale); restaurato ff. sciolti, utilizzabili in parte.

Peyron-Tamburini (M.III.1-9)

M.III.15 Mémoires sur toutes les actions de guerre.

Cart., sec. XVII, mm 288 x 180, ff. 27 frammentari, di cui 1 bianco (in origine pp. $90+$ indice). Bruciato in profondità nelle parti dorsali con distruzione di testo; reperiti 2 ff. iniziali e quelli finali, utilizzabili in minima parte.

Peyron-Tamburini (M.III.15)

M.IV.16 Histoire abregée de cinq proposition par le sieur Le Chars, 1692.

Cart., sec. XVII, mm 190/235 x 85/130, ff. 201 frammentari (bianchi 1 all'inizio e 3 alla fine). Gravemente danneggiato dal fuoco che lo arse in profondità sui margini anteriori con parziale distruzione del testo; restaurato a ff. sciolti, utilizzabili in minima parte.

Peyron-TAmburini (M.IV.16)

M.IV.29-30 Institution d'un Prince ou Traitté des qualitez, des vertus et des devoirs d'un souverain divisé en quatre parties - Tome II: Des devoirs du Prince par rapport au gouvernement temporal; ... Tome III: Des qualitez personelles et des vertus d'un Prince chretien.

Cart., sec. XVIII, mm 245 x 175, Tome II: ff. 160; Tome III: ff. 145. Bruciati sulle zone marginali con perdita delle glosse, andarono scompaginati; restaurati a ff. sciolti sono utilizzabili in gran parte.

Peyron-Tamburini (M.IV.28-30)

M.V.11 Les Ethiques d'Aristote à son filz Nichomachus.

Cart., sec. XVI-XVII, mm 154 x 165, ff. 44 frammentari. Bruciato completamente nella parte superiore, andò scompaginato; restaurato a ff. sciolti, utilizzabili in minima parte.

Peyron-Tamburini (M. V.11)

M.V.13 [Inni o Chansons per il Natale, in francese e in latino].

Cart., sec. XVI, mm 225 x 170, ff. 33 frammentari con vari disegni a penna. Bruciato sui dorsi e sui margini anteriori e superiori, con parziale perdita di testo, andò scompaginato; leggibile in discreta parte. Peyron-Tamburini (M.V.13)

M.V.19 Ecrits sur le formulaire.

Cart., sec. XVII-XVIII, mm 205/225 x 140/160, ff. 313, di cui 7 iniziali con titolo, indice e 3 non scritti (in origine pp. 790). Danneggiato nelle zone marginali dal fuoco, con parziale perdita di testo; restaurato a ff. sciolti utilizzabili in gran parte. 
Peyron-Tamburini (M.V.19)

M.V.20 [?] Mémoires sur la vie et la prison de Monsieur l'abbé de Saint Cyran, dressées par Monsieur Lancelot, de l'ordre de Monsieur de Sacy.

Cart., sec. XVII, mm 200/230 x 140/165, ff. 77 in parte frammentari (originariamente pp. 173). Bruciato nelle zone marginali, con parziale perdita di testo (nella maggior parte dei ff. le infiltrazione d'acqua non asciugate aggravarono lo stato di conservazione, facendo anche sbiadire le scritture); andò smembrarto e disperso in mezzo ai frammenti da identificare. Le parti reperite, restaurate a ff. sciolti, sono utilizzabili in minima parte.

Peyron-Tamburini (M.V.20)

M.V.21 Abrégé de l'bistoire de la Royale Maison de Savoie.

Cart., sec. XVIII, mm 180/220x 140/155, ff. 64 frammentari. Bruciato sui dorsi e sui margini, andò scompaginato e disperso in mezzo ai frammenti da identificare. La parte reperita è quella iniziale: utilizzabile solo in parte a causa dei danni del fuoco.

Peyron-Tamburini (M.V.21)

M.VI.6 Comédie de Monsieur de Bussy[-Rabutin]; L'bistoire de Madame et de Monsieur le Comte de Guiche...

Cart., sec. XVII, mm 192/204 x 160, ff. 56. Danneggiato dal fuoco sui margini superiori con lieve consunzione di testo; restaurato a ff. sciolti leggibili in gran parte.

Peyron-Tamburini (M.VI.6)

M.VI.7 [Ms. miscellaneo contenente opuscoli vari di poesia e di medicina, tra cui:] Tractatus de virtutibus berbarum (in francese).

Cart., sec. XV, mm 180 x 120, ff. 6 frammentari (da unire alle parti identificate da Vinay e da Vitale-Brovarone, già restaurate a ff. sciolti): testo utilizzabile solo in parte a causa dei rilevanti danni provocati dal fuoco e dall'acqua di spegnimento.

Mss. Saluzzo f. 24r; App. al Pasini f. 241r s.v. Tractatus; Giacosa p. 511; Peyron-Tamburini (M.VI.7); Vinay nº 99; Vitale-Brovarone p. 465

Bc. 68-69 Alexandre de Saluces, Histoire de la Milice Piemontaise et des guerres du Piemont depuis l'an 1536 au 1747.

Cart., sec. XVIII, mm 285 x 185, ff. 15 (uniti in occasione del restauro alle restanti parti identificate da Vitale-Brovarone). Bruciati sulle zone marginali, andarono scompaginati in mezzo ai frammenti da identificare; restaurati a ff. sciolti, utilizzabili in parte.

App. al Pasini f. 240v s.v. Saluces; Vitale-Brovarone p. 467

Bc. 96

Discours sur toutes les affaires qui sont aujourdhuy entre les Maisons de France et d'Autriche.

Cart., sec. XVII, mm 250 x 175, 1 f. (ultimo del testo), da unire a tutti gli altri identificati da Vitale-Brovarone e successivamente restaurati sciolti.

App. al Pasini f. 228r s.v. Discours 
Bc. 113 [?] Traité de paix et alliance entre le roy François I et les Cantons Suisses; [seguono trattati con altri re di Francia e duchi di Savoia fino al 1764].

Cart., sec. XVIII, mm 170/200 x 95/160, ff. 160 in parte frammentari (di cui 4 bianchi in fine). Danneggiato dal fuoco che consunse la parte iniziale con distruzione parziale di testo nei ff. rimasti. Restaurato a ff. sciolti utilizzabili in parte.

App. al PAsini f. 241r s.v. Traité

Bc. 121 Antoine Purqueddu, Le trésor de la Sardaigne dans les vers a soie, poème traduit en 1789 par G. M. Simon.

Cart., sec. XVIII, mm 110/135 x 75/90, ff. 2 frammentari (da unire alle parti identificate da Vitale-Brovarone), notevolmente bruciati con distruzione di testo: utilizzabili in minima parte.

App. al Pasini f. 237v s.v. Purqueddu; Vitale-Brovarone p. 465 (per un refuso segnatura indicata come Bc. 12)

* $1^{a}$ S.R. 162 Interruption du sommeil Cabalistique ou le Dévoilement des Tableaux (ora K.V.12) Mystiques de l'Antiquité...

Cart., sec. XVII-XVIII, mm 322 x 215, cc. 118. Si tratta della prima parte del ms. $1^{\text {a }}$ S.R. 162, andato smembrato dopo l'incendio, il cui titolo non è presente nella Appedice al Pasini (vi compaiono invece le altre parti identificate da Vitale-Brovarone: Pratique sur le vin et le tartre...; Traité de la Philosophie Chymique du petit paysan... par Balachie...). La provenienza da un unico ms. - oltre che dall'identità della tipologia dei danni - è provata dalla presenza dell'annotazione "Chapitre 13" sul f. 1r, che fa riferimento alla nota di possesso "Marchese di Rivarolo" riportata all'inizio del $13^{\circ}$ capitolo del primo trattato identificato da Vitale-Brovarone. Testo completamente utilizzabile.

App. al PAsini f. 237r s.v. Pratique, f 226r s.v. Balachie; Cosentini n ${ }^{\circ}$ 1610 (K.V.12); Vitale-Brovarone p. 467 (1ª delle Sale Riservate 162) 